Homology, Homotopy and Applications, vol.10(2), 2008, pp.257-272

\title{
PRECOVERS, LOCALIZATIONS, AND STABLE HOMOTOPY
}

\author{
M. GRIME \\ (communicated by J. P. C. Greenlees)
}

\begin{abstract}
We prove a new localization theorem for algebraic stable categories when the localizing subcategory is generated by a precovering class in the model category. We use this to show how one may explicitly realize certain Bousfield localization functors that arise naturally in the study of relative homological algebra of groups.
\end{abstract}

\section{Introduction}

The results of this paper were originally formulated in order to provide an explicit description of certain Bousfield localization functors in modular representation theory. The original motivation was in attempting to generalize Rickard's construction of idempotent modules, [Ric97], to other triangulated categories arising from relative cohomology theories for group algebras.

Let us state our localization result, which appears as a corollary of Theorem 3.2. If the reader is unfamiliar with any of the terms, then he is encouraged to read Section 2, where we will explain our conventions.

Theorem. Let $\mathcal{E}$ be a Frobenius category with triangulated quotient $\mathcal{T}$, and let $\mathcal{R}$ be a class of objects in $\mathcal{E}$. Suppose that the following hypotheses are satisfied:

(i) $\mathcal{R}$ is a precovering class in $\mathcal{E}$ and all objects of $\mathcal{E}$ have finite $\mathcal{R}$-dimension;

(ii) $\mathcal{R}$ is closed under shifts in $\mathcal{T}$;

(iii) for all $R$ in $\mathcal{R}$ the injective hull $I(R)$ is in $\mathcal{R}$.

Let $\mathcal{S}$ be the localizing subcategory $\langle\mathcal{R}\rangle^{\oplus} \subseteq \mathcal{T}$; then the quotient functor, $j: \mathcal{T} \rightarrow$ $\mathcal{T} / \mathcal{S}$, when it exists, has a right adjoint $j !$.

We believe it is worth remarking on the link with the notion of a homotopy colimit. Suppose that $\mathcal{E}$ is, in fact, abelian so that we may think of short exact sequences rather than conflations. If $X$ can be written as a countable filtered direct limit in $\mathcal{E}$, then

$$
X \cong \underset{n \in \mathbb{N}}{\lim _{n}} X_{n}
$$

Received October 30, 2007, revised December 9, 2007, May 21, 2008; published on November 17, 2008.

2000 Mathematics Subject Classification: 18E10, 18E30, 18E35, 18G25, 20J05, 55U35.

Key words and phrases: homology, homotopy, localizations, stable categories, exact categories.

This article is available at http://intlpress.com/HHA/v10/n2/a13

Copyright (c) 2008, International Press. Permission to copy for private use granted. 
with $X_{n}$ in $\mathcal{R}$, and if the natural short exact sequence

$$
0 \rightarrow \oplus X_{i} \rightarrow \oplus X_{i} \rightarrow X \rightarrow 0
$$

is an $\mathcal{R}$-resolution of $X$, then $j ! j X$ is related to the homotopy colimit of $X_{n}$ via the triangle

$$
\operatorname{hocolim} X_{n} \rightarrow X \rightarrow j ! j X \rightarrow
$$

in $\mathcal{T}$. Note that if we assume the exact structure on $\mathcal{E}$ is given by all short exact sequences, then there is actually an isomorphism in $\mathcal{T}$

$$
\operatorname{hocolim}\left(X_{i}\right) \rightarrow X
$$

but in general this is not the case.

In order to prove that $j$ ! exists, in greater generality, we will demonstrate the existence of a triangle

$$
X_{\mathcal{R}} \rightarrow X \rightarrow X_{\mathcal{R}^{\perp}} \rightarrow
$$

with $X_{\mathcal{R}} \in\langle\mathcal{R}\rangle^{\oplus}$, and $\operatorname{Hom}_{\mathcal{T}}\left(\mathcal{R}, X_{\mathcal{R}^{\perp}}\right)=0$. Let us call such an object the localization triangle of $X$. It follows that $j ! j X \cong X_{\mathcal{R}^{\perp}}$.

We now explain the structure of the paper. Section 2 will provide an explanation of the definitions and conventions adopted throughout the paper.

In Section 3 we will show how, given an object $X$ in $\mathcal{E}$ and a finite resolution by of objects in $\mathcal{R}$, one may pass to the quotient $\mathcal{T}$ to construct something that we will label a homotopy approximation. We spend the bulk of the section demonstrating that an approximation yields an object $L_{0}(X)$ which, with the hypotheses we place on $\mathcal{R}$, satisfies

$$
\operatorname{Hom}_{\mathcal{T}}\left(R, L_{0}(X)\right) \cong \operatorname{Hom}_{\mathcal{T}}(R, X)
$$

for all $R$ in $\mathcal{R}$.

We explain in Section 4 how one may use the results of Section 3 to show the existence of adjoints to inclusion and quotient functors arising from the localizing subcategory $\mathcal{R}$ generates and the localizing subcategory of $\mathcal{R}$-local objects. The reader should note that, despite the original motivation coming from Bousfield localizations, we do not need any hypotheses on compact generation.

These results are then applied, in Section 5, to the relatively stable categories of a group algebra. This is the application that originally motivated our construction. In general, the relatively stable category need not be generated by the finite dimensional modules. Thus the finite dimensional modules (compactly) generate a proper localizing subcategory. The quotient functor has a right adjoint by Bousfield. Note that we obtain the same as the localizing subcategory by considering the pure projective modules in the module category: a pure projective module is an arbitrary direct sum of indecomposable finite dimensional modules. If we assume all modules have finite pure projective dimension (say if the group algebra is countable as a set), then our results provide an alternate proof that the quotient functor exists, and an explicit construction of it in a finite number of steps.

To conclude this introduction, we will explain the ideas behind the results in Section 3. The proof of Theorem 3.2 is quite lengthy and formal, yet it encapsulates a 
straightforward idea which we explain here. Recall that we seek a triangle

$$
X_{\mathcal{R}} \rightarrow X \rightarrow X_{\mathcal{R}^{\perp}} \rightarrow
$$

with $X_{\mathcal{R}}$ enjoying a unique lifting property; for every object $R \in \mathcal{R}$ and morphism

$$
R \rightarrow X
$$

in $\mathcal{T}$, there should be a unique factorization in $\mathcal{T}$ through

$$
X_{\mathcal{R}} \rightarrow X
$$

Given the assumption that $\mathcal{R}$ is precovering, then for any object $X$ in $\mathcal{E}$ there is a precover in $\mathcal{E}$

$$
R_{X} \rightarrow X
$$

and in $\mathcal{E}$ every map from an object in $\mathcal{R}$ to $X$ factors through $R_{X}$ in $\mathcal{E}$. Thus if we pass to $\mathcal{T}$ and complete this map to a triangle

$$
R_{X} \rightarrow X \rightarrow Y \rightarrow
$$

we would have a plausible candidate for the desired localization triangle in $\mathcal{T}$. However, the uniqueness of the lifting does not follow: we know we may lift to $\mathcal{E}$ to obtain $a$ factorization, but we also know that it will be far from unique. To correct our naïve initial guess, we should make an adjustment to kill these extra maps. Suppose that the map

$$
R_{X} \rightarrow X
$$

has a kernel $K$ in $\mathcal{E}$ and furthermore that $K$ is in $\mathcal{R}$. On passing back to $\mathcal{T}$ one can complete the map $K \rightarrow R_{X}$ to a triangle

$$
K \rightarrow R_{X} \rightarrow X_{\mathcal{R}} \rightarrow \text {. }
$$

Since the composite

$$
K \rightarrow R_{X} \rightarrow X
$$

is zero, we can complete

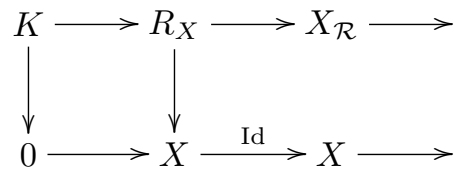

to a morphism of triangles; whence we deduce a map

$$
X_{\mathcal{R}} \rightarrow X,
$$

which we complete to a triangle

$$
X_{\mathcal{R}} \rightarrow X \rightarrow X_{\mathcal{R}^{\perp}} \rightarrow
$$

This turns out to be the localization triangle we seek. The reader will have spotted that we have assumed $K$ is in $\mathcal{R}$, when it need not be, and in general has no reason to be. However, if $X$ has a finite $\mathcal{R}$-dimension, then $K$ has dimension one smaller, and this draws the reader to looking for some inductive argument to side-step this issue. We shall present such an argument in proving Theorem 3.2. 


\section{Preliminary material}

The reader is encouraged to consult [Hap92] or [Kel96] for a more thorough explanation, or [HPS97] for a topological point of view. The conventions we adopt are algebraic, rather than topological. Thus we will refer to Frobenius categories with stable quotient, inflations and deflations (definition to follow) rather than model and stable homotopy categories, fibrations and cofibrations.

First, we note that if $\mathcal{C}$ is any category, we will use

$$
(X, Y)_{\mathcal{C}}
$$

for the set of morphisms from $X$ to $Y$ in $\mathcal{C}$. We will write greek letters to represent morphisms.

An exact category is an additive $k$-linear category equipped with a class of distinguished pairs of morphisms $(\iota, \rho)$, called conflations (we refer to $\iota$ as an inflation and $\rho$ as a deflation). We require that $\iota$ is a kernel of $\rho, \rho$ is a cokernel of $\iota$, and they satisfy the axioms:

- the identity morphism of the zero object is a deflation;

- the composition of two deflations is a deflation;

- given a deflation $\rho: U \rightarrow V$ and a morphism $Y \rightarrow V$ there is a pullback diagram

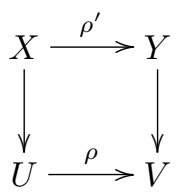

with $\rho^{\prime}: X \rightarrow Y$ a deflation,

and the dual axioms for inflations. Modulo some set theoretic concerns, one can think of exact categories as full subcategories of a module category and conflations are a subclass of short exact sequences.

Given an exact category, an object $P$ is projective if, for any deflation $\rho: X \rightarrow Y$, the induced map

$$
\rho_{*}:(P, X)_{\mathcal{E}} \rightarrow(P, Y)_{\mathcal{E}}
$$

is epic. Dually, $I$ is injective if, for each inflation $\iota: U \rightarrow V$, the map

$$
\iota^{*}:(V, I)_{\mathcal{E}} \rightarrow(U, I)_{\mathcal{E}}
$$

is epic. We may now define a Frobenius category.

Definition 2.1. A category $\mathcal{E}$ is Frobenius if it is an exact category, the classes of injective and projective objects coincide, and for all $X$ there is a deflation

$$
P(X) \rightarrow X
$$

and an inflation

$$
X \rightarrow I(X,)
$$

and both $P(X)$ and $I(X)$ are in the category of pro/injective objects. We refer to $P(X)$ as a projective cover of $X$, and $I(X)$ as an injective hull of $X$. 
Examples of Frobenius categories include the module category of a symmetric algebra (e.g., a group algebra), and the category of chain complexes over an abelian category, where conflations are short exact sequences of complexes that are split in each degree.

There is an important theorem about Frobenius categories that is due to Happel, [Hap92].

Theorem 2.2. Let $\mathcal{E}$ be Frobenius, and let $\mathcal{P}$ be the class of projective/injective objects; then the quotient

$$
\mathcal{T}:=\frac{\mathcal{E}}{\mathcal{P}}
$$

is a triangulated category.

A Frobenius category $\mathcal{E}$ is thus an example of a model category, and the quotient $\mathcal{T}$ is its homotopy category; see [HPS97] for details.

It remains for us to define the notion of a precovering class.

Definition 2.3. Let $\mathcal{E}$ be an exact category. A class of objects $\mathcal{R} \subseteq \mathcal{E}$ is precovering if for each $X$ in $\mathcal{E}$ there is an object $R_{X}$ in $\mathcal{R}$ and a morphism

$$
R_{X} \rightarrow X
$$

called a precover of $X$ with the property that any morphism $R \rightarrow X$ with $R \in \mathcal{R}$ factors through $R_{X} \rightarrow X$.

We comment that there is nothing necessarily unique about $R_{X}$ nor the morphism. Moreover, the astute reader will notice that if $\mathcal{E}$ is in fact Frobenius, then the projective objects are an example of a precovering class.

We now fix a Frobenius category $\mathcal{E}$, with triangulated quotient $\mathcal{T}$ and precovering class $\mathcal{R}$.

Definition 2.4. The category $\mathcal{E}$ has enough kernels with respect to $\mathcal{R}$ if every precover

$$
R_{X} \rightarrow X
$$

has a kernel.

If $\mathcal{E}$ has enough kernels with respect to $\mathcal{R}$, let $K$ be the kernel of $R_{X} \rightarrow X$. We can now take a precover of $K$, take its kernel, and so on to create an $\mathcal{R}$-resolution of $X$. The reader should note that we are not assuming that the triple

$$
K_{i+1} \rightarrow R_{i} \rightarrow K_{i}
$$

is a conflation in $\mathcal{E}$.

It is worth inserting some examples at this point to illustrate the kinds of areas where we wish to apply our ideas.

Example 2.5. We give two examples using module categories.

- Let $\mathcal{E}$ be $\bmod (k G)$ of a finite group algebra, and let $H$ be a subgroup of $G$. A module is $H$-projective if it is a summand of $M \otimes \operatorname{Ind}_{H}^{G}(k)$ for some $M$. The 
class of all $H$-projective modules is precovering, and moreover a precover of $X$ is

$$
\operatorname{Ind}_{H}^{G}\left(\operatorname{Res}_{H}^{G}(X)\right) \rightarrow X,
$$

with the map coming from the counit of the adjunction. Note $\mathcal{E}$ is abelian so we have enough kernels.

- Let $\mathcal{E}$ be the category $\operatorname{Mod}(A)$ of all modules for some finite dimensional Frobenius algebra $A$ (i.e. one whose module category is a Frobenius category, again $k G$ is a concrete example). Let $\mathcal{R}$ be the class of modules isomorphic to (possibly infinite) direct sums of finite dimensional objects. This is the class of pure projective modules; it is precovering. If $M$ is written as the direct limit of its finite dimensional submodules in $\mathcal{E}$,

$$
M:=\underline{\lim _{\longrightarrow}} M_{i},
$$

then a precover is given by the natural map

$$
\coprod_{i \in I} M_{i} \rightarrow M .
$$

We need one more definition pertaining to $\mathcal{R}$.

Definition 2.6. Let $X$ be in $\mathcal{E}$ and suppose that

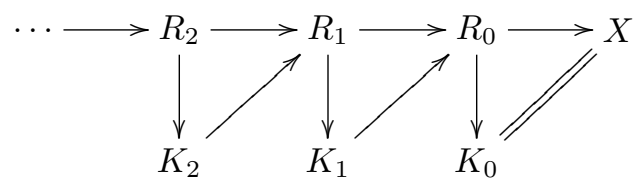

is an $\mathcal{R}$-resolution. If $K_{d}$ is in $\mathcal{R}$ for some $d$, then we say $X$ has finite $\mathcal{R}$-dimension, and if $d$ is minimal among all $\mathcal{R}$-resolutions we say $X$ has $\mathcal{R}$-dimension $d$. If no such $d$ exists then $X$ has infinite $\mathcal{R}$-dimension. We will write $\operatorname{dim}_{\mathcal{R}}(X)=d$ to indicate the $\mathcal{R}$-dimension.

We will finish this section by explaining our conventions for triangulated categories. We assume the reader is familiar with the theory of triangulated categories. If not, then we recommend the introduction to [Nee02]. If $\mathcal{T}$ is triangulated, and $X$ is an object of $\mathcal{T}$, then we will write $X[1]$ for the right shift functor. This is suspension to the topologist, and the inverse Heller translate to a representation theorist. If $\alpha: X \rightarrow Y$ is a map in $\mathcal{T}$, then we write

$$
X \stackrel{\alpha}{\longrightarrow} Y \longrightarrow \operatorname{cone}(\alpha) \longrightarrow
$$

for the (strictly speaking, a) corresponding distinguished triangle in $\mathcal{T}$. In our examples, where $\mathcal{T}$ is a quotient of $\mathcal{E}$, triangles are in one-to-one correspondence with conflations, and the shift functor is realized by the conflation in $\mathcal{E}$

$$
X \rightarrow I(X) \rightarrow X[1]
$$

for $I(X)$ an injective hull of $X$. 
Definition 2.7. A full triangulated subcategory $\mathcal{S} \subseteq \mathcal{T}$ is localizing if it is closed under $\mathcal{T}$-direct sums; i.e., if $X_{i}$ is a set indexed collection of objects in $\mathcal{S}$, and the coproduct

$$
\coprod_{i \in I} X_{i}
$$

exists in $\mathcal{T}$, then it is in $\mathcal{S}$. If $\mathcal{R}$ is an collection of objects in $\mathcal{T}$, we write

$$
\langle\mathcal{R}\rangle^{\oplus}
$$

for the smallest localizing subcategory containing $\mathcal{R}$ (the intersection of all localizing subcategories containing $\mathcal{R}$ ). The localizing subcategory $\mathcal{R}^{\perp}$ of $\mathcal{R}$-local objects is the full subcategory of objects, $X$, satisfying

$$
(R[n], X)_{\mathcal{T}}=0
$$

for all $R \in \mathcal{R}$ and $n \in \mathbb{Z}$.

Definition 2.8 (Verdier localization). Let $\mathcal{S}$ be a localizing subcategory of $\mathcal{T}$. The Verdier localization $\mathcal{T} / \mathcal{S}$ is the category obtained by formally adjoining the inverses of all morphisms $\alpha$ such that cone $(\alpha)$ is in $\mathcal{S}$.

More formally, maps in the localization are equivalence classes of roofs. Given $\alpha$, $\beta$ in $\mathcal{T}$ such that cone $(\alpha)$ is in $\mathcal{S}$, the equivalence class $\left[\beta \alpha^{-1}\right]$ is represented by

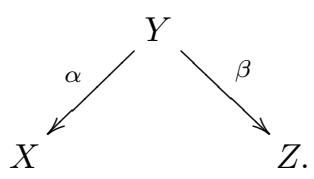

Two roofs, $X \leftarrow Y \rightarrow Z$ and $X \leftarrow Y^{\prime} \rightarrow Z$, lie in the same equivalence class if there is an object $Y^{\prime \prime}$ and isomorphisms $Y^{\prime \prime} \rightarrow Y, Y^{\prime \prime} \rightarrow Y^{\prime}$ in $\mathcal{T} / \mathcal{S}$ in which the obvious diagrams commute.

We make one observation about the set theoretic issues involved. Morphisms are equivalence classes of such diagrams, and a priori there is no reason to suppose that the equivalence classes are sets. We will not concern ourselves with this question in this note, and we shall assume that all constructions create sets within the same universe as $\mathcal{T}$.

\section{Triangulated categories}

\subsection{Finite resolutions}

We continue with the hypotheses that $\mathcal{E}$ is Frobenius with triangulated quotient $\mathcal{T}, \mathcal{R}$ is a precovering class in $\mathcal{E}$ and that all objects have $\mathcal{R}$ resolutions. Let $X$ be any object in $\mathcal{E}$, and suppose further that the $\mathcal{R}$-dimension of $X$ is finite. We know that there is a diagram with all morphisms and objects considered in $\mathcal{E}$ :
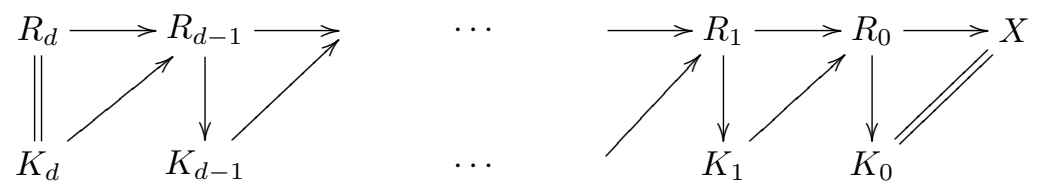
and in each subdiagram,

$$
K_{i+1} \rightarrow R_{i} \rightarrow K_{i}
$$

$R_{i}$ is a precover of $K_{i}$, and $K_{i+1}$ is its kernel.

We now pass to the quotient $\mathcal{T}$ and construct a homotopy approximation of the resolution in $\mathcal{T}$, starting from the left by defining a sequence of maps $\epsilon_{i}$, and objects $L_{i}$ in $\mathcal{T}$. First, we define $\epsilon_{d}$ to be the map $R_{d} \rightarrow R_{d-1}$. Now we will construct $\epsilon_{d-1}$. By assumption, the composite

$$
R_{d} \rightarrow R_{d-1} \rightarrow K_{d-1}
$$

is zero in $\mathcal{E}$, so there is a diagram

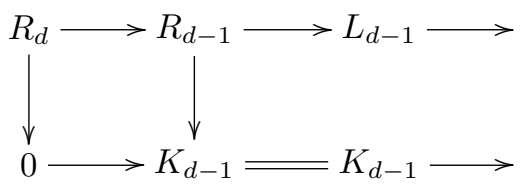

in which the rows are distinguished triangles in $\mathcal{T}$. We complete to a morphism of triangles

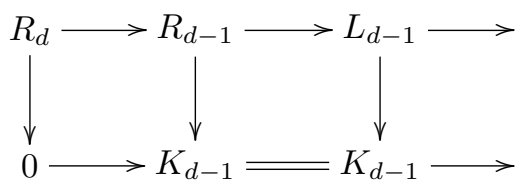

and define $\epsilon_{d-1}$ to be the composite

$$
L_{d-1} \longrightarrow K_{d-1} \longrightarrow R_{d-2} .
$$

Define $L_{d-2}$ to be the cone of $L_{d-1} \rightarrow R_{d-2}$. We note that by construction the composite $L_{d-1} \rightarrow R_{d-2} \rightarrow K_{d-2}$ is also zero, so we obtain another morphism of triangles

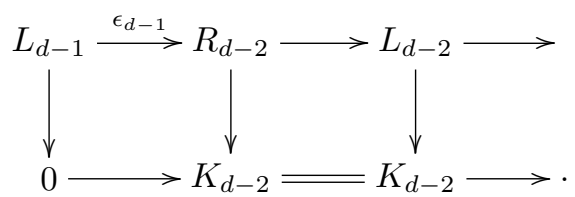

Now we define $\epsilon_{d-2}$ as the composite $L_{d-2} \rightarrow K_{d-2} \rightarrow R_{d-3}$. We can iterate this process and we will end up with a diagram

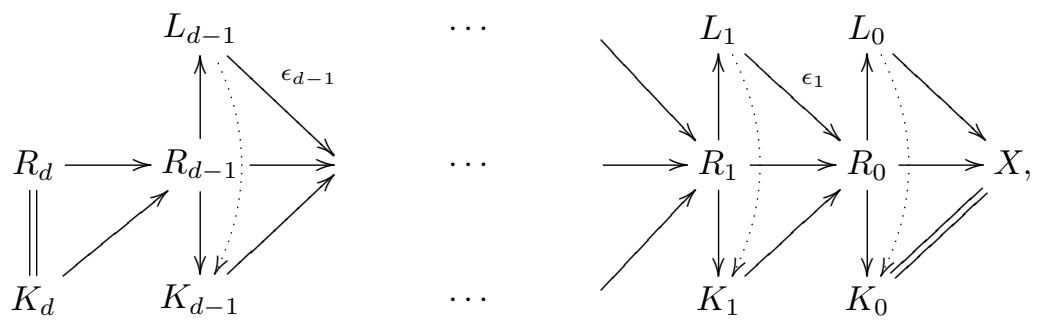

where all objects and morphisms are in $\mathcal{T}$. If we wish to emphasise the dependence on $X$, we will also write $L_{0}(X)$, for $L_{0}$. 
Remark 3.1.

(i) $L_{0}$ is unique in $\mathcal{T}$, but, a priori, not up to unique isomorphism.

(ii) It lies in the smallest subcategory that contains $R_{i}$ for $0 \leqslant i \leqslant d$ and is closed under triangles.

(iii) If all of the triples $K_{i} \rightarrow R_{i-1} \rightarrow K_{i-1}$ are isomorphic to distinguished triangles, then $L_{0}$ is stably isomorphic to $X$.

(iv) If $X$ is the direct limit of a sequence of objects, $X_{i}$ in $\mathcal{R}$, and moreover the natural short exact sequence defining $X$ :

$$
0 \rightarrow \oplus X_{i} \rightarrow \oplus X_{i} \rightarrow X \rightarrow 0
$$

is an $\mathcal{R}$-resolution, then $L_{0}$ is the homotopy colimit

$$
\operatorname{hocolim}\left(X_{i}\right)
$$

in $\mathcal{T}$.

(v) We genuinely needed the model structure to make this construction: one can define precovers in a triangulated category, however, a triangulated category does not have any non-trivial kernels. The best one could hope for is a diagram,

$$
R_{d} \longrightarrow R_{d-1} \longrightarrow \cdots \longrightarrow R_{1} \longrightarrow R_{0} \longrightarrow X
$$

in which the composite of two consecutive morphisms is zero. Whilst one could certainly start the construction, one would quickly find that attempts to construct a map from $L_{d-2}$ to $R_{d-3}$ are doomed to failure.

\subsection{The main theorem}

We are now in a position to state our first theorem. It asserts that under some mild, but slightly lengthy hypotheses, the object $L_{0}$ has the unique lifting property.

Theorem 3.2. Let $\mathcal{E}$ be a Frobenius category with triangulated quotient $\mathcal{T}$, and let $\mathcal{R}$ be a class of objects in $\mathcal{E}$. Suppose that the following hypotheses are satisfied:

(i) $\mathcal{R}$ is a precovering class in $\mathcal{E}$;

(ii) $\mathcal{R}$ is closed under shifts in $\mathcal{T}$;

(iii) for all $R$ in $\mathcal{R}$ the injective hull $I(R)$ is in $\mathcal{R}$.

Further suppose that $X$ is an object in $\mathcal{E}$ with finite $\mathcal{R}$-dimension $d$ and suppose that $L_{0}$ is constructed as above; then there is an isomorphism $\left(R, L_{0}\right)_{\mathcal{T}} \cong(R, X)_{\mathcal{T}}$ for all $R$ in $\mathcal{R}$.

We will break the proof down into a series of straightforward lemmas. First we deal with surjectivity.

Lemma 3.3. The map $\left(R, L_{0}\right)_{\mathcal{T}} \rightarrow(R, X)_{\mathcal{T}}$ in Theorem 3.2 is surjective. 
Proof. Let $R \rightarrow X$ be any morphism in $\mathcal{T}$. Lift arbitrarily to a morphism in $\mathcal{E}$. This must factor through the precover $R_{0} \rightarrow X$. Recall that the ultimate step in our construction yielded a morphism of triangles

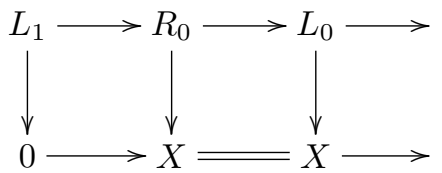

from which we deduce that the lift $R \rightarrow R_{0} \rightarrow X$, when we pass back to $\mathcal{T}$, factors as $R \rightarrow R_{0} \rightarrow L_{0} \rightarrow X$.

Thus it remains to show that the map is an injection. The proof of injectivity is more complicated. First, we will need a standard observation about how one may choose to factor maps in $\mathcal{T}$.

Lemma 3.4. With the hypotheses (i) and (iii) of Theorem 3.2, let $R$ be in $\mathcal{R}$ and consider a map $\alpha: R \rightarrow X$ in $\mathcal{E}$. By assumption, this factors in $\mathcal{E}$ as

$$
R \stackrel{\beta}{\longrightarrow} R_{0} \stackrel{\gamma}{\longrightarrow} X
$$

since $R_{0}$ is a precover. Suppose that $\alpha$ is 0 in the quotient $\mathcal{T}$, then there is a map $\delta: R \rightarrow R_{0}$ in $\mathcal{E}$ such that:

1. the composite $\gamma \delta$ is zero in $\mathcal{E}$;

2. $\delta=\beta$ in $\mathcal{T}$.

Proof. Since $\alpha=0$ in $\mathcal{T}$ it factors as $R \rightarrow I(R) \rightarrow X$. The assumption that $I(R)$ is in $\mathcal{R}$ is key as this means that the map $I(R) \rightarrow X$ factors through $R_{0}$. Set $\delta^{\prime}$ to be the composite $R \rightarrow I(R) \rightarrow R_{0}$. This is the amount by which we need to correct $\beta$; thus we define $\delta:=\beta-\delta^{\prime}$.

We prove the next lemma by making one more assumption, and then we shall prove that the assumption is true.

Lemma 3.5. Suppose that $\alpha$ is in the kernel of the map $\left(R, L_{0}\right)_{\mathcal{T}} \rightarrow(R, X)_{\mathcal{T}}$ and further assume that $\alpha$ factors through $R_{0}$ in $\mathcal{T}$; then $\alpha=0$.

Proof. This is best illustrated diagrammatically — it is a straightforward argument, but an algebraic proof would overload the reader with notation. We start with a diagram

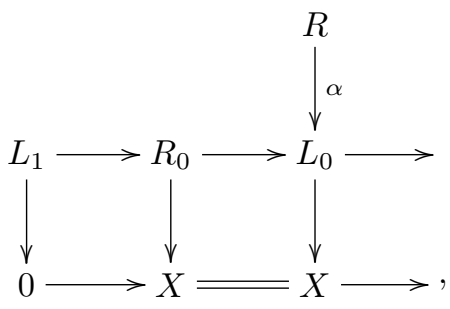

where the rows are distinguished triangles. Our extra assumption that $\alpha$ factors 
through $R_{0}$ allows us to create a commutative diagram

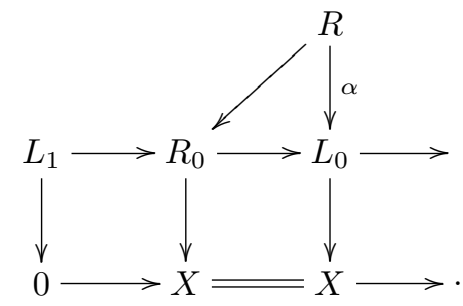

An elementary diagram chase shows that the map $R \rightarrow R_{0} \rightarrow X$ is zero in $\mathcal{T}$. Thus we my invoke Lemma 3.4, and deduce that we can choose it to be zero in $\mathcal{E}$. Thus the map $R \rightarrow R_{0}$ must factor in $\mathcal{E}$, and thus in $\mathcal{T}$, through $K_{1}$. Since $R$ is in $\mathcal{R}$, and we actually have a map in $\mathcal{E}$ from $R$ to $K_{1}$, this factors, again in $\mathcal{E}$ through $L_{1}$, which is a precover of $K_{1}$. Thus we have a larger commutative diagram

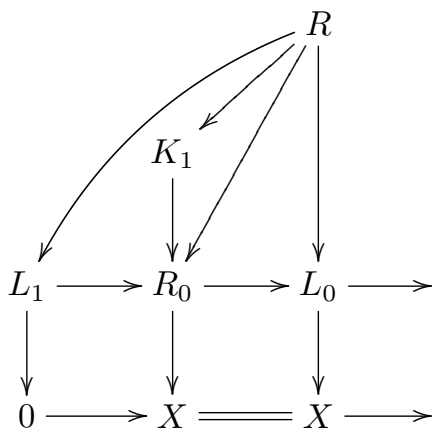

and we see that $\alpha$ factors through two consecutive maps in a distinguished triangle, and must be 0 in $\mathcal{T}$.

This leaves the extra assumption in Lemma 3.5 to be proven. The proof inducts on the $\mathcal{R}$-dimension.

Lemma 3.6. Any map from $R \rightarrow L_{0}$ factors through $R_{0}$ in $\mathcal{T}$.

Proof. The statement is equivalent to the assertion that the natural map

$$
\left(R, R_{0}\right)_{\mathcal{T}} \rightarrow\left(R, L_{0}\right)_{\mathcal{T}}
$$

is surjective. First we deal with the case of $\mathcal{R}$-dimension 0 . This is just the case that $X$ is in $\mathcal{R}$, but then any $\mathcal{R}$-resolution, after removing summands, is isomorphic (in the category of chain complexes over $\mathcal{E}$ ) to

$$
\cdots \rightarrow 0 \rightarrow 0 \rightarrow X \rightarrow X
$$

and there is nothing to prove since $L_{0}=X$. To prove the general case consider the 
three maps in $\mathcal{T}$

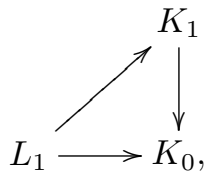

where by induction on $\mathcal{R}$-dimension we may assume that $L_{1} \rightarrow K_{1}$ has the property that every map from an object in $\mathcal{R}$ to $K_{1}$ factors uniquely through it since $K_{1}$ has $\mathcal{R}$-dimension one smaller than $K_{0}=X$. For example, we may assume that the induced map

$$
\left(R, L_{1}\right)_{\mathcal{T}} \rightarrow\left(R, K_{1}\right)_{\mathcal{T}}
$$

is an isomorphism. We invoke the octahedral axiom to construct

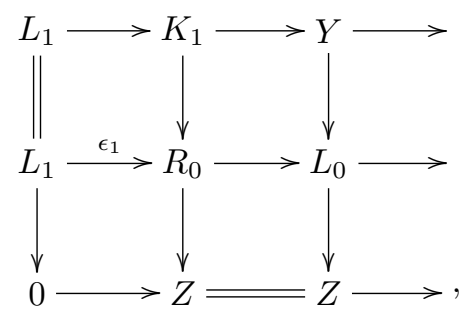

where by induction on $\mathcal{R}$-dimension again, we have that $(R, Y)_{\mathcal{T}}=0$. Apply the functor $(R, ?)_{\mathcal{T}}$ to obtain

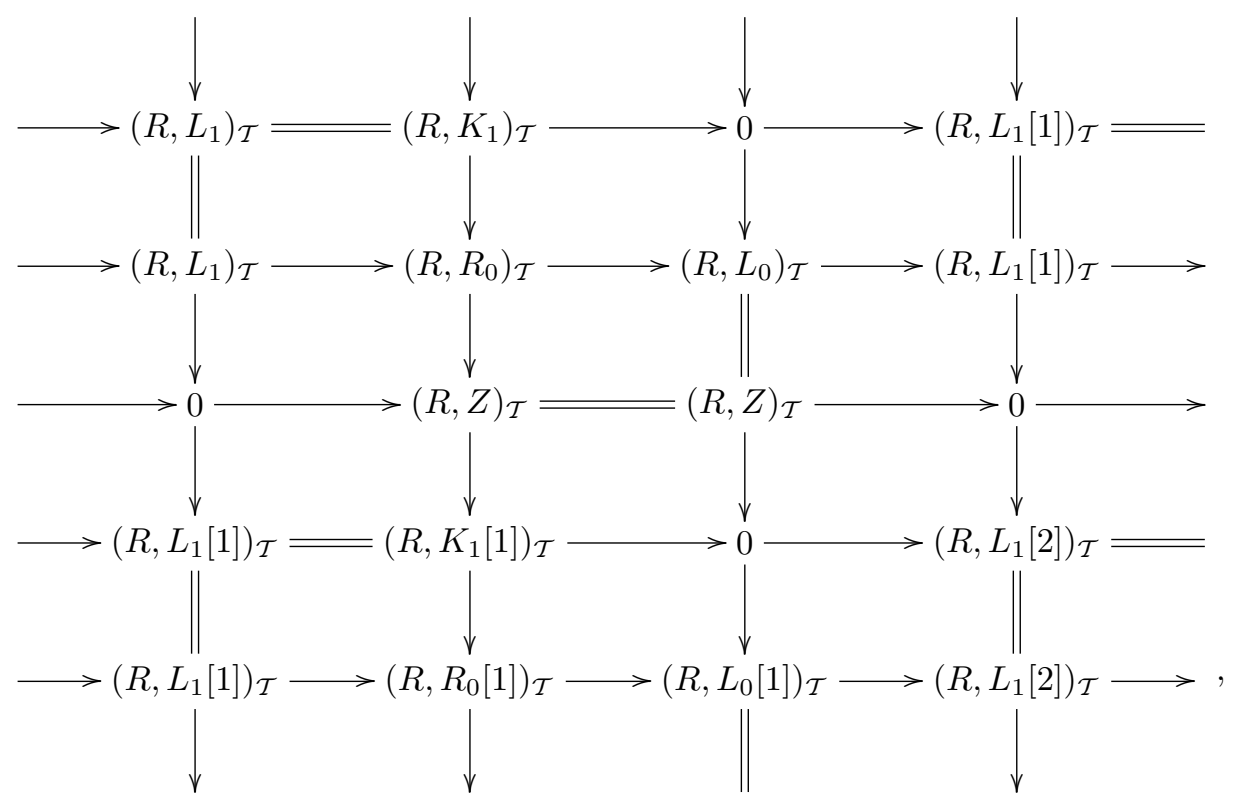

where all rows and columns are exact. There is a standard diagram chase to be done. 
The following statements are equivalent:

(i) $\left(R, R_{0}\right)_{\mathcal{T}} \rightarrow\left(R, L_{0}\right)_{\mathcal{T}}$ is surjective.

(ii) $\left(R, R_{0}\right)_{\mathcal{T}} \rightarrow(R, Z)_{\mathcal{T}}$ is surjective.

(iii) $(R, Z)_{\mathcal{T}} \rightarrow\left(R, K_{1}[1]\right)_{\mathcal{T}}$ is zero.

(iv) $\left(R, K_{1}[1]\right)_{\mathcal{T}} \rightarrow\left(R, R_{0}[1]\right)_{\mathcal{T}}$ is injective.

(v) $\left(R, K_{1}\right)_{\mathcal{T}} \rightarrow\left(R, R_{0}\right)_{\mathcal{T}}$ is injective.

To prove the last of these conditions, we work in $\mathcal{E}$. Suppose that $R \rightarrow K_{1} \rightarrow R_{0}$ is zero; then there is a diagram in $\mathcal{E}$,

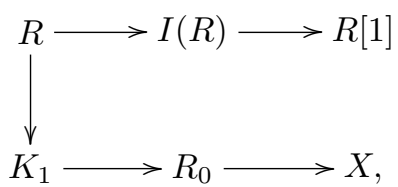

and the top row is a conflation. By assumption there is a map $I(R) \rightarrow R_{0}$ which makes

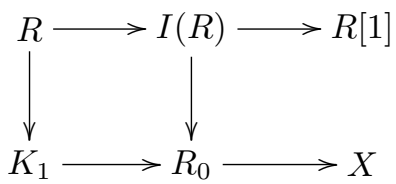

commutative. This can be completed to

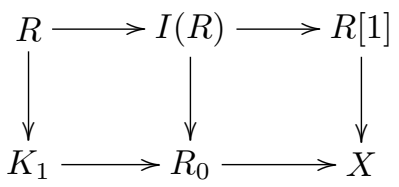

since $I(R) \rightarrow R[1]$ is a cokernel of $R \rightarrow I(R)$. There is one hypothesis yet to be used, which we now invoke. As $\mathcal{R}$ is closed under shifts, the map from $R[1] \rightarrow X$ factors through $R_{0}$, which implies that the map $I(R) \rightarrow R_{0}$ factors through $K_{1}$; hence $R \rightarrow$ $K_{1}$ factors through $I(R)$ as we were required to show.

Lemmas 3.3, 3.4, 3.5 and 3.6 complete the proof of the main theorem.

\section{Constructing adjoints}

Suppose that $\mathcal{E}, \mathcal{T}$, and $\mathcal{R}$ satisfy the hypotheses of Theorem 3.2 . We can use this to deduce the existence of adjoints to certain inclusion functors via a routine argument.

Theorem 4.1. Suppose that every object in $\mathcal{T}$ has finite $\mathcal{R}$-dimension. Let $\mathcal{S}$ be the smallest full localizing subcategory of $\mathcal{T}$ that contains $\mathcal{R}$; then the inclusion functor

$$
i: \mathcal{S} \rightarrow \mathcal{T}
$$

has a right adjoint $i^{!}$. 
Proof. Given $X$ in $\mathcal{T}$ we may construct $L_{0}$ as above. Define $i^{!}(X):=L_{0}(X)$. Notice that for $R \in \mathcal{R}$, Theorem 3.2 gives isomorphisms.

$$
(i(R), X)_{\mathcal{T}} \cong\left(R, L_{0}(X)\right)_{\mathcal{T}} \cong\left(R, L_{0}(X)\right)_{\mathcal{S}} \cong\left(R, i^{!}(X)\right)_{\mathcal{S}}
$$

Thus we just need to argue that these isomorphisms exist if we replace $R$ with an arbitrary object $S$ in $\mathcal{S}$. It is clear that the class of objects for which the isomorphisms exist contains $\mathcal{R}$ and is closed under direct sums and triangles; hence it contains $\mathcal{S}$, and we are done.

Corollary 4.2. If the quotient $j: \mathcal{T} \rightarrow \mathcal{T} / \mathcal{S}$ exists, then it has a right adjoint, $j$ !

Proof. Define $j_{!}(X)$ to be the third object in the triangle

$$
L_{0}(X) \rightarrow X \rightarrow Y
$$

There is clearly a theorem to be stated if we drop the assumption on finite $\mathcal{R}$-dimension of all objects.

Theorem 4.3. Suppose that $\mathcal{E}, \mathcal{T}$, and $\mathcal{R}$ are as in Theorem 3.2. Let $\mathcal{S}$ be the smallest localizing subcategory containing $\mathcal{R}$. If the inclusion

$$
i: \mathcal{S} \rightarrow \mathcal{T}
$$

has a left adjoint, $i^{!}$, and if $X$ has finite $\mathcal{R}$-dimension, then necessarily $i^{!}(X)$ is isomorphic to the $L_{0}(X)$ coming from our construction.

\section{Applications in relative homological algebra}

We should attempt to convince the reader that the assumptions we have placed on $\mathcal{E}$ are really the kinds of things she might meet in everyday mathematics. The original motivation for this construction came from modular representation theory.

Let $A$ be a finite dimensional algebra over a field $k$. Suppose that $\mathcal{P}$ is some class of objects in $\operatorname{Mod}(A)$ such that the quotient

$$
\frac{\operatorname{Mod}(A)}{\mathcal{P}}
$$

is triangulated. An example would be if $A$ were Frobenius, and $\mathcal{P}$ the class of all projective/injective objects. However, this is far from being the only interesting exact structure we may put on a module category, as we shall see shortly.

It is natural to ask how the (isomorphism classes of) finite dimensional objects behave inside this category. Thus we define

$$
\mathcal{R}:=\operatorname{Add}(\bmod (A))
$$

as the class of pure projective objects. This is precovering; see [HJ07] for example. The smallest triangulated subcategory containing $\mathcal{R}$ coincides with the smallest triangulated subcategory containing the finite dimensional objects that is closed under direct sums. Again, we shall use $\mathcal{S}$ to denote this triangle closure of $\mathcal{R}$. We can now cite some examples of uses of the localization theorems. 
Example 5.1. If every module has finite pure projective dimension, then the inclusion $\mathcal{S} \rightarrow \mathcal{T}$ has a right adjoint by Theorem 4.1.

Example 5.2. If the inclusion has an adjoint, then we can calculate it using Theorem 4.3 for those objects with finite pure projective dimension.

Examples of finite pure projective dimension abound, as we now explain. The precise implications depend on one's set theory.

1. If the cardinality of the underling set of $A$ is $\aleph_{t}$ for some finite ordinal $t$, then every module has pure projective dimension at most $t+1$. If the cardinality is finite then every module has pure projective dimension 1.

2. For an algebra $A$ over any field $k$, if the $k$-dimension of a module $M$ is $\aleph_{t}$, then $M$ has pure projective dimension at most $t+1$.

The reader is referred to [HJ07, (3.8)] for more examples. We will finish by fleshing out the bones of these examples. As we have mentioned, the original motivation comes from modular representation theory.

Suppose that $G$ is a finite group, $k$ a field of characteristic $p$, and that $p$ divides $|G|$. Let $H$ be a subgroup of $G$. Define $\mathcal{P}$ to be the class of summands of all modules induced up from $H$. It is now classical that $\operatorname{Mod}(k G) / \mathcal{P}$, the relatively stable category, is triangulated. The triangles correspond to short exact sequences that split on restriction to $H$. One recovers the normal notion of projective, and the usual stable category $\operatorname{StMod}(k G)$, by letting $H$ be the trivial subgroup.

One can define Rickard modules in $\operatorname{Mod}(k G) / \mathcal{P}$ given a compactly generated subcategory; however, the relative case is fundamentally different from the usual stable module category. It is a simple exercise to show that the smallest triangulated subcategory of $\operatorname{StMod}(k G)$ that contains the simple modules and is closed under direct sums is $\operatorname{StMod}(k G)$. It is known, [Gri06, Ch. 7 and Appl. B], that the finite dimensional objects may (compactly) generate a proper subcategory of the relatively stable category, and thus yield a non-trivial localization functor. Our construction gives an explicit description of it, under certain conditions:

Example 5.3. If $|k|=\aleph_{t}$, then all modules have finite pure projective dimension, since $|k G|=\aleph_{t}$. This is no real restriction, since there are countable algebraically closed fields, and this is more than sufficient for modular representation theory.

Example 5.4. If $k$ is arbitrary, then one can apply Theorem 4.3 since the inclusion has an adjoint, as we have observed.

\section{Acknowledgements}

I would like to thank Peter Jørgensen for commenting on the general case, and in particular for correcting an earlier erroneous proof of Lemma 3.6, as well as explaining the content of [HJ07] and its implications for the construction presented here.

This work was funded (in part) by the Heilbronn Institute for Mathematical Research. 


\section{References}

[Gri06] M. Grime, Triangulated categories, adjoint functors and Bousfield localization, Ph.D. thesis, University of Bristol, 2006.

[Hap92] D. Happel, On the derived category of a finite-dimensional algebra, Commentarii Mathematici Helvetici 62, no. 3 (1992), 339-389.

[HJ07] H. Holm and P. Jørgensen, Compactly generated homotopy categories, Homology, Homotopy Appl. 9, no. 1 (2007), 257-274.

[HPS97] M. Hovey, J.H. Palmieri, and N.P. Strickland, Axiomatic stable homotopy theory, Mem. Amer. Math Soc. 128, no. 610, 1997, 114pp.

[Kel96] B. Keller, Derived categories and their uses, in Handbook of algebra, Vol. 1, pp. 671-701 (M. Hazewinkel, ed.), Elsevier, Amsterdam (1996).

[Nee02] A. Neeman, Triangulated categories, Annals of Math. Studies 148, Princeton University Press, Princeton, NJ (2001).

[Ric97] J. Rickard, Idempotent modules in the stable category, J. London Math. Soc. 56, no. 1 (1997), 149-170.

[Ric00] J. Rickard, Bousfield localization for representation theorists, Infinite length modules (Bielefeld, 1998), Trends in Math., 273-283, Birkhäuser, Basel (2000).

M. Grime matt.grime@gmail.com

School of Mathematics, University of Bristol, University Walk, Bristol, BS8 1TW, United Kingdom 